\title{
Cornell University Library: Books by Q.H. Vuong
}

Library Catalog

Access:

March 2, 2021

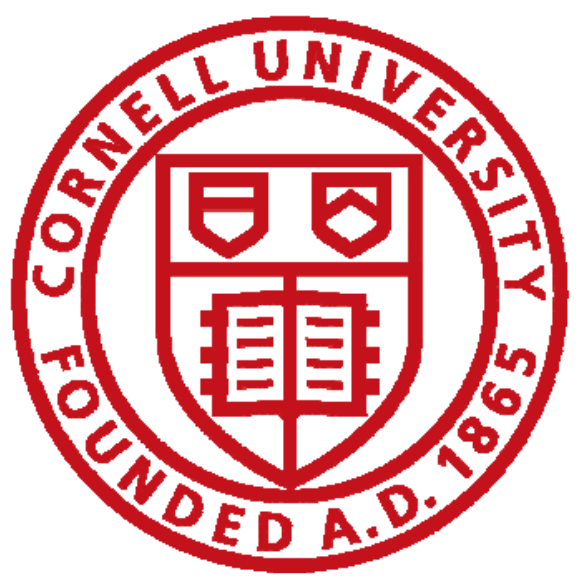

Cornell University

Querying URL:

https://newcatalog.library.cornell.edu//?f\%5Bformat\%5D\%5B\%5D=Book\&q=Quan\%20H oang\%20Vuong\&search_field=all_fields

The search results in three books by Q.H. Vuong, which follow.

1. Financial markets in Vietnam's transition economy : facts, insights, implications

Call number: HG187.V5 V86 2010 (Kroch Library Asia); URL:

https://newcatalog.library.cornell.edu/catalog/10328931

2. Kinh tế Việt Nam: thăng trầm và đột phá

Call number: HC444 .P517 2009 (Kroch Library Asia); URL:

https://newcatalog.library.cornell.edu/catalog/6678816

3. Bằng chứng cuộc sống: suy nghĩ về phát triển bền vững Việt Nam

Call number: HC444.Z9 E5 2015; URL:

https://newcatalog.library.cornell.edu/catalog/9379144 
Hybrid library for a hybrid semester: COVID-19 updates »

\section{LIBRARY CATALOG}

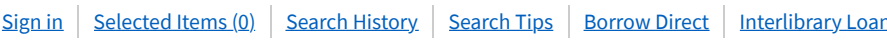

Search...

Q ADVANCED SEARCH $\mid$ ? ASKA LIBRARIAN $\mid$ MY ACCOUNT

B Back to catalog results (10328931)

$$
\square \text { Select } \quad \nabla \text { Cite } \rightarrow \text { Export } \theta \text { Print }
$$

\section{Financial markets in Vietnam's transition economy}

facts, insights, implications

Quan-Hoang Vuong.

Availability
Kroch Library Asia Hours/Map
HG187. V5 V86 2010 DText

$\checkmark$ Available

\begin{tabular}{l|l} 
Request item & Request scan of article/chapter
\end{tabular}

Author, etc.:

Vương,Quân Hoàng: Info

Format:

E Book

Language:

English.

Published:

Saarbrücken : VDM Verlag Dr. Müller, 2010.

Subject:

Finance $>$ Vietnam.

Financial institutions $>$ Vietnam.

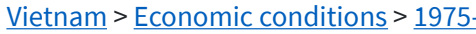

Description:

xx, 369, lii pages : illustrations, charts ; $22 \mathrm{~cm}$

ISBN:

3639233832

9783639233834

Notes:

Includes bibliographical references (pages xxxv-xlix) and index.

Browse related items by call number

HG187.V5 V86 2010 


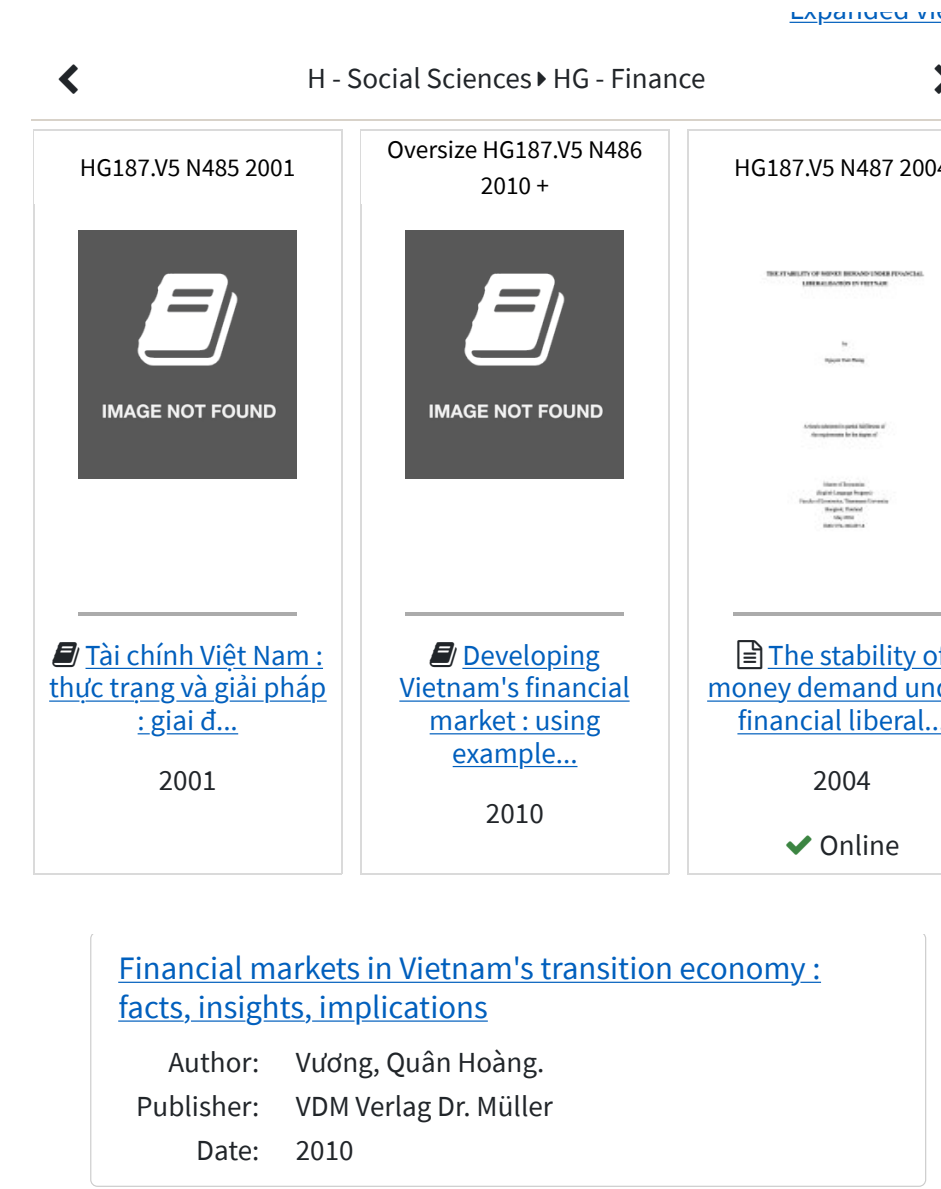

Librarian View

CONTACT US

Feedback

? Ask a Librarian

RESOURCES

Library Website Search Catalog Articles \& Full Text Databases E-journal Titles

Release Notes

Images 


\section{LIBRARY CATALOG}

\begin{tabular}{l|l|l|l|l} 
Sign in Selected Items (0). & Search History & Search Tips & Borrow Direct & Interlibrary Loan
\end{tabular}

Search...

Q ADVANCED SEARCH $\mid$ ? ASKA LIBRARIAN $\mid$ I MYACCOUNT

○ Back to catalog results $(6678816)$

$$
\square \text { Select } \quad \nabla \text { Cite } \rightarrow \text { Export } \rightarrow \text { Print }
$$

\section{Kinh tếViệt Nam}

thăng tràm và đột phá

Phạm Minh Chính, Vương Quân Hoàng.

Availability

Kroch Library Asia Hours/Map

HC444 .P517 2009 DText

Available

\begin{tabular}{l|l} 
Request item Request scan of article/chapter \\
\hline
\end{tabular}

Author, etc.:

Phạm, Minh Chính. Info

Format:

早 Book

Language:

Vietnamese.

Published:

Hà Nội : Nhà xuát bản Chính trị quốc gia, 2009.

Subject:

Economic development $>$ Vietnam $>$ History.

Structural adjustment (Economic policy) $>>$ Vietnam $>$ History.

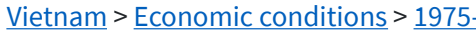

Summary:

Portraits of strategy and development of economic in Vietnam.

Description:

xxx, 554 p. : ill. ; $23 \mathrm{~cm}$.

Other contributor:

Vương, Quân Hoàng.

Notes:

Includes bibliographical references (p. 539-549) and index.

Browse related items by call number 
H - Social Sciences HC - Economic History \& Conditions HC94-1085 - By region or country

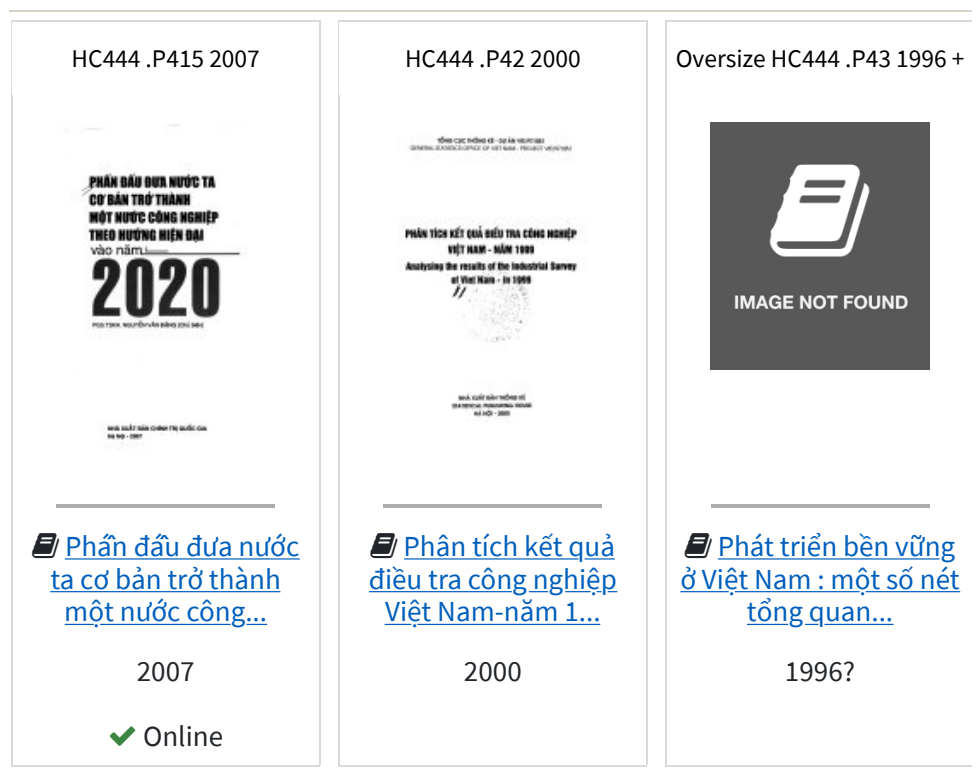

Kinh tế Việt Nam : thăng trâm và đột phá

Author: Phạm, Minh Chính.

Publisher: Nhà xuát bản Chính trị quốc gia

Date: 2009

Librarian View

CONTACT US

? Ask a Librarian

Feedback

RESOURCES

Release Notes

Library Websit

Search

Catalog

Articles \& Full Text

Databases

E-journal Titles

Credits

Images 


\section{LIBRARY CATALOG}

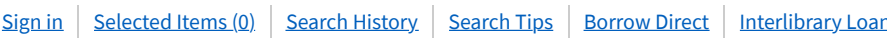

Search...

Q ADVANCED SEARCH $\mid$ ? ASKA LIBRARIAN $\mid$ \& MYACCOUNT

- Back to catalog results (9379144)

$$
\square \text { Select } \boldsymbol{D} \text { Cite } \boldsymbol{\nabla} \text { Email } \rightarrow \text { Export } \boldsymbol{\theta}
$$

\section{Bằng chứng cuộc sống}

suy nghĩ về phát triển bền vững Việt Nam

Bạch Ngọc Chiến, Vương Quân Hoàng.
All Fields

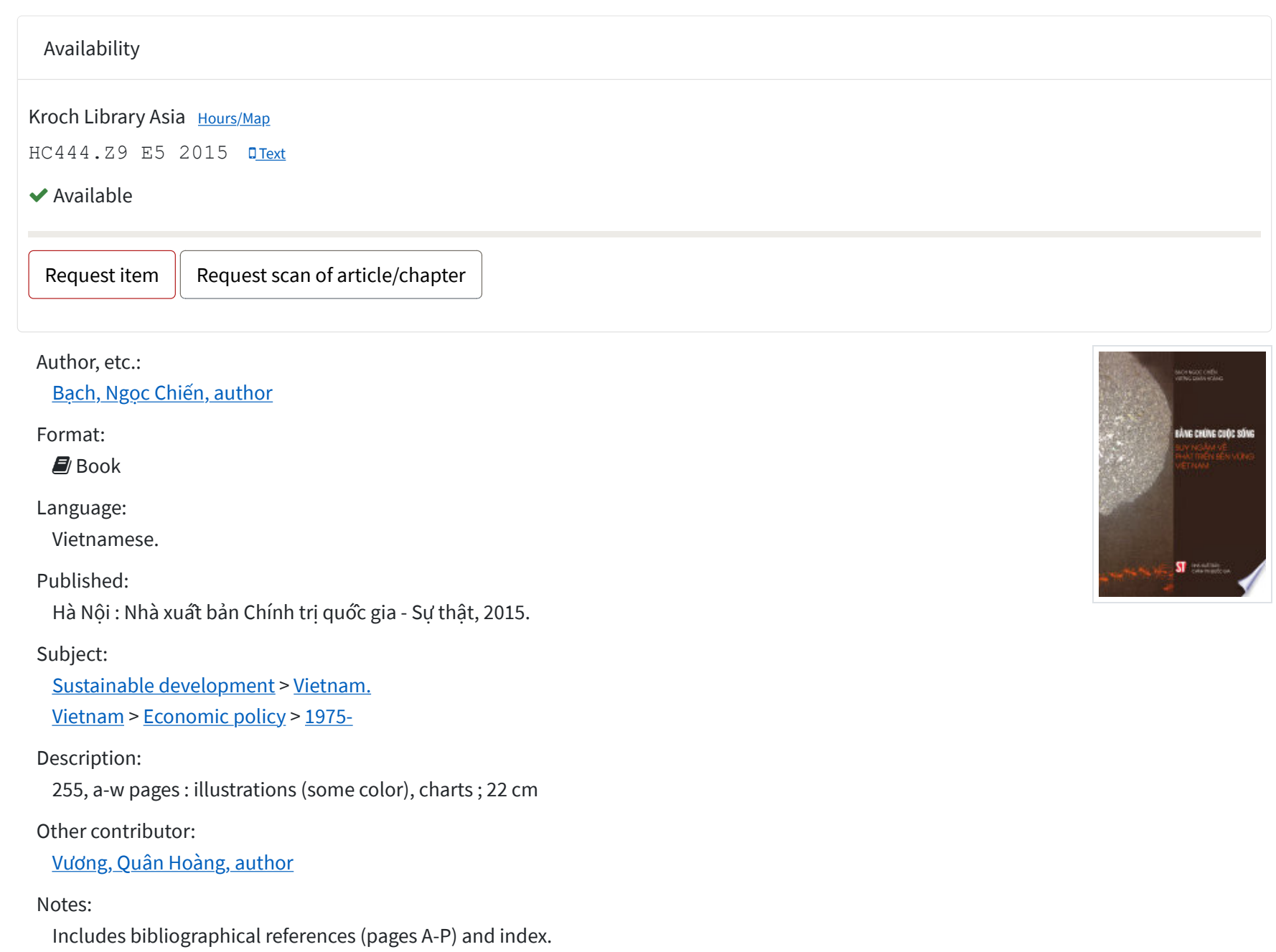

Browse related items by call number

HC444.29 E5 2015

Expanded view 


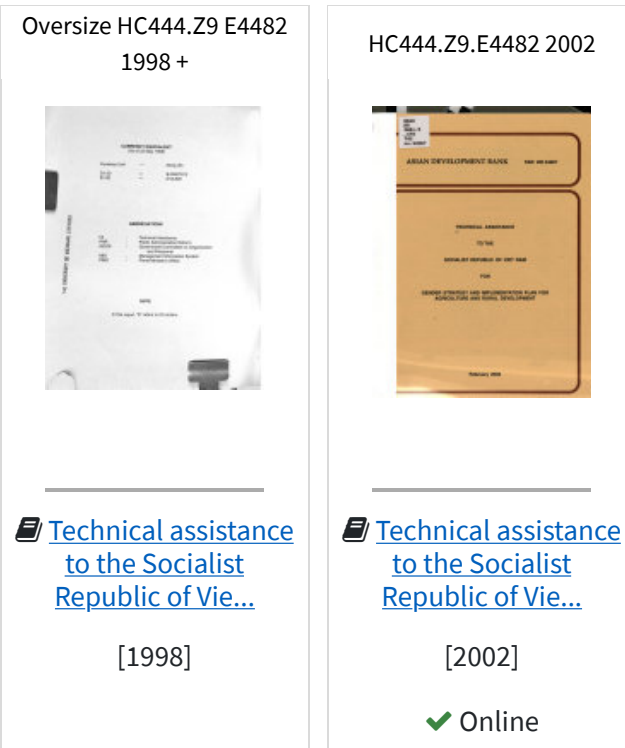

HC444.Z9 E4483 1997

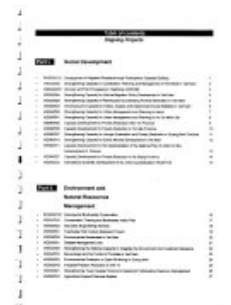

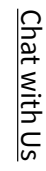

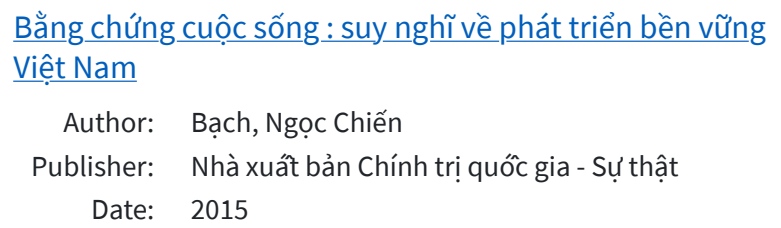

Librarian View

CONTACT US

? Ask a Librarian

\section{Feedback}

RESOURCES

LibraryWebsite Search Catalog Articles\&Full Text Databases E-journal Titles

$\underline{\text { Release Notes }}$

Images 


\section{References:}

[1] Quan-Hoang Vuong. (2010). Financial markets in Vietnam's transition economy. VDM Verlag, Germany.

[2] Phạm Minh Chính, Vương Quân Hoàng. (2009). Kinh tế Việt Nam: Thăng trầm và Đột phá. Nxb Chính trị Quốc gia, Hà Nội.

[2] Bạch Ngọc Chiến, Vương Quân Hoàng. (2000). Bằng chứng cuộc sống: Suy nghĩ về phát triển bền vững Việt Nam. Nxb Chính trị Quốc gia, Hà Nội. 